\title{
A Arte do Repente e as Habilidades Lingüisticas*
}

\section{Antonio Roazzi}

Universidade Federal de Pernambuco (UFPE)

\section{Ann Dowker Peter}

\section{E. Bryant}

Universidade de Oxford

Investiga a relação entre as habilidades lingüisticas e a arte do repente, em sujeitos repentistas (violeiros ou cantadores), poetas orais encontrados no Nordeste do Brasil, muitos dos quais têm uma escolaridade limitada. Vinte repentistas e dezoito não-repentistas com faixa etária, escolaridade e nível sócio-econômico semelhantes foram examinados em um conjunto de tarefas envolvendo produção de rimas, segmentação fonológica, memória de listas de palavras e velocidade de leitura. Os repentistas produziram um número de rimas quatro vezes superior aos não-repentistas, além de apresentarem uma velocidade superior na produção da primeira rima. Nas outras tarefas, os dois grupos de sujeitos não diferiram de modo significativo. Assim, a habilidade dos repentistas parece ser ao mesmo tempo altamente desenvolvida e especializada, Estas descobertas corroboram o ponto de vista de que possam, haver dissociações entre rima e algumas outras habilidades lingüísticas, como a segmentação fonológica, assim como aparentemente não existe uma relação direta entre a educação formal $e$ o desenvolvimento de habilidades culturalmente informais.

\section{Introdução}

A imagem convencional de um poeta é a de uma pessoa altamente letrada e educada, que se senta a escrever versos, com

\footnotetext{
* Várias instituições contribuíram para a realização desta pesquisa. Mais especificamente, os autores agradecem ao INEP e ao CNPq, por terem financiado a coleta de dados no Brasil, e ao Conselho Britânico e à British Academy, por terem financiado a fase de análise
} 
uma prolongada e cuidadosa reflexão. A poesia, de acordo com essa visão, é, predominantemente, uma forma escrita; as suas características orais são secundárias. Apesar disso, há muitos poetas que compõem e apresentam seus poemas oralmente, mesmo sendo completamente incapazes de ler e escrever ou fazendo-o de modo muito limitado, o que, ainda assim, não os impede de criar poesia altamente sofisticada. Este estudo envolve um grupo desses poetas.

A existência de tais poetas orais tem sido geralmente ignorada pelos psicólogos, embora Morais, Alegria e Content (1987) tenham chamado a atenção para a existência de poetas iletrados e argumentado que estes são a prova de que a consciência para a rima, como tal, independe do domínio da leitura. Talvez a não-referência a esses poetas se deva ao fato de a sua existência ser considerada natural, já que os jogos de palavras que envolvem invenções poéticas e a composição de versos orais parecem ser atividades importantes para muitas pessoas. A rima é uma atividade universal, mas, como muitas outras atividades comuns, ainda é uma parte mal compreendida da vida humana. Encontramos rimas nos slogans políticos, em jingles de advertência, na linguagem rimada do cockney, em motes, mnemônicas e piadas e, é claro, em poemas e canções.

E bastante claro que muitas pessoas consideram as rimas interessantes e divertidas. As crianças também demonstram apreciar a rima na forma de canções e cantigas de ninar, desde a mais tenra idade (MacLean, Bryant e Bradley, 1986), podendo-se fazer julgamentos seguros acerca do assunto desde, pelo menos, os três

de dados na Inglaterra. Agradecemos também ao Dr. Lynette Bradley, da Universidade de Oxford, pelas valiosas discussões nos estágios iniciais da investigação; à $\operatorname{Dr}^{\mathrm{a}}$ Terezinha Nunes, por ter facilitado a realização da pesquisa, colocando à disposição a infra-estrutura necessária no Brasil, e ao Dr. Robert Hiorns, da Universidade de Oxford; às Dr ${ }^{\text {as }}$ Joyce Snell e Lynda White, do Colégio Imperial da Universidade de Londres, pela assessoria estatística. Enfim, gostaríamos de agredecer, também, à pesquisadora Shirley Cristina Lacerda Malta, pela valiosa contribuição à realização da pesquisa, durante a fase de coleta de dados. 
anos de idade (Lenel e Canter, 1981; Bryant e Bradley, 1985), e as próprias crianças gostam de produzir rimas e outros artifícios poéticos (por exemplo, a aliteração), em alguns casos desde a idade de dois anos (Luckens, 1984; Ghukovsky 1968; Schwartz, 1977; Dowker, 1989). Foi demonstrado, inclusive, (Schieffelin, 1983) que, mesmo em uma comunidade papua, onde os adultos desencorajam os jogos infantis com sons, as crianças são indulgentes com esse tipo de jogo, quando brincam umas com as outras.

Sendo a composição de poemas bastante comum nas crianças pré-letradas (Chukovsky, 1968; Dowker, 1989), é de se esperar que também seja entre os adultos analfabetos e semi-alfa-betizados (Finnegan, 1977 e 1978). Estratégias fonológicas são comuns nesse tipo de poesia. Elas incluem a aliteração (um importante componente da épica do Kazaquistão, da poesia mongol e da poesia clássica da Somália, da mesma forma que o foi na poesia oral em inglês antigo), a dassonância (comum, por exemplo, na poesia dos índios americanos) e a rima (comum, por exemplo, na poesia birmanesa, turca, fidjiana, assim como, é claro, na maioria da poesia oral européia e, numa menor extensão, na poesia escrita).

Apesar da ubiqüidade da rima e de sua óbvia predileção por parte das pessoas em geral, os psicólogos, até recentemente, prestaram pouca atenção ao fenômeno, especialmente em relação a crianças mais velhas e adultos. Na maioria das pesquisas que têm sido feitas, a óbvia preferência das pessoas por rimas tem sido usada como um artifício para descobrir alguma outra coisa. Assim, o fato de que as pessoas confundem palavras que rimam, nas tarefas de memória, tornou-se um ponto de importância nas pesquisas nessa área (Conrad, 1971; Baddeley, 1978; Hulme, 1984). Da mesma forma, em trabalhos que estabelecem uma forte relação entre a sensibilidade das crianças à rima e o seu sucesso no aprendizado da leitura (Bradley e Bryant, 1983), é geralmente pressuposto que essas tarefas de rima simplesmente medem a consciência fonológica. Assim, as pesquisas com rimas produziram modelos de memória, no primeiro caso, e hipóteses acerca da rela- 
ção entre rima e aprendizagem da leitura, no outro. Nenhum tipo de pesquisa lidou com a rima como tal, em qualquer nível.

Contudo, algumas pesquisas recentes têm mostrado que as crianças são capazes de lidar com a rima e a aliteração (vide acima) muitos anos antes de conseguirem cumprir tarefas nas quais elas tenham de detectar ou segmentar fonemas isolados (Bruce, 1964; Liberman, Schankweiler, Liberman, Fowler e Fisher, 1977; Nesdale, Herriman e Tummer, 1984). Lundberg, Frost e Peterson (1988, p.282) concluíram de seus estudos com crianças pequenas que, enquanto o treinamento direto melhora o desempenho das crianças no último tipo de tarefa, o rimar não depende muito do ensino, porque envolve "menor consciência e deliberada manipulação dos segmentos". Considerando que as crianças geralmente não têm um bom desempenho em tarefas que envolvam a detecção ou deleção de fonemas isolados, até os sete anos de idade, aproximadamente, tem sido afirmado que as crianças só se tornam capazes de resolver essas tarefas como resultado do aprendizado da leitura (por exemplo, Bruce, 1964; Morais, Cary, Alegria e Bertelson, 1979). Essa discrepância entre as aptidões para lidar com rimas e com fonemas sugere que a habilidade de rimar não pode ser considerada simplesmente como uma dentre várias habilidades fonológicas.

Existem evidências mais profundas de que as aptidões para lidar com rimas e com fonemas não são fortemente correlacionadas. Em um estudo de Stanovich, Cunningham e Cramer (1984) não foi encontrada forte relação entre os testes de rima e outras medidas de habilidade fonológica em crianças no início da escolaridade (em parte porque os testes de rima eram muito mais fáceis para as crianças). Parte de outra pesquisa recente (Bryant, MacLean, Bradley e Crossland, 1990) indica que a ligação entre rima e leitura é independente da relação entre outros testes fonológicos e leitura. Parece que a rima dá a sua própria e distinta contribuição para o aprendizado da leitura, que não é a mesma da consciência dos fonemas (para uma revisão da literatura e uma discussão mais detalhada sobre este assunto, ver Beech e 
Colley 1987; Bertelson, Gelder, Tfouni e Morais, 1989; Morais, 1991; Roazzi, 1991 e 1992; Roazzi e Dowker, 1989).

Neste trabalho nós observamos um grupo de pessoas notadamente especializadas em rimas, com o objetivo de investigar a extensão de sua habilidade e até que ponto essa habilidade está ou não relacionada com outras aptidões, ou com a experiência educacional. Estas pessoas são os repentistas, poetas orais encontrados no Nordeste do Brasil.

Uma notável característica dos repentistas (Tavares, 1982) é a extrema aderência aos detalhes e às regras complexas da rima e da métrica. Esses poetas se engajam em competições cantadas, onde lhes pode ser solicitado pela audiência que usem qualquer uma de um grande número de possíveis formas poéticas, cada uma das quais é precisamente definida em termos de esquema de rima, número de sílabas por verso, acentuação etc. Por exemplo, a sextilha consiste em seis versos de sete sílabas com o esquema de rima $\mathrm{ABCBDB}$; a décima consiste em dez versos de sete sílabas com o esquema de rima AABAACCDDA; o quadrão consiste em oito versos de sete sílabas com o esquema de rima $\mathrm{AAABCCCB}$; o martelo tem o mesmo esquema de rima que a décima mas os versos têm dez sílabas cada um. Além disso, os poemas devem fazer sentido e muitas vezes devem lidar com um tópico particular "mote" — dado pela audiência. Muitas vezes o mote consiste em dois versos e os repentistas podem criar glosas (poemas que trabalham com tópicos predeterminados) com versos que rimam com os do mote. Assim, os limites fonológicos e semânticos são rigorosos. Além disso, os repentistas não têm tempo para pensar, planejar e rever as suas composições, o que é um direito dos poetas convencionais. Apesar disso, tal poesia é, muitas vezes, de alta qualidade. Nas palavras do repentista Dimas Batista:

Eu muito admiro o poeta de praça Que passa dois meses fazendo um quarteto No fim de um ano é que acaba um soneto E quando termina inda fica sem graça; Com tinta e papel o esboço ele traça Contando nos dedos pra metrificar; 
Que noites de sono ele perde a pensar Para apresentar tão minguado produto Que desses eu faço dois, três, em um minuto Cantando galope na beira do mar."

As habilidades marcantes dos repentistas são um fenômeno interessante por si só. Mas elas também nos dão a oportunidade única de explorar a relação entre a rima e outras habilidades. Poder-se-ia prever que os repentistas se sairiam melhor que os outros em um teste objetivo de produção de rimas, mas seria interessante descobrir o quão melhor e também se os repentistas diferem, de outros modos, dos não-repentistas. Por exemplo, a sua experiência e proficiência com as rimas significam que eles também são superiores em segmentação fonética? Considerando a literatura nessa área e as especulações acerca da relação entre as aptidões fonológicas e a leitura (por exemplo, Bryant e Bradley, 1985; Morais, Alegria e Content, 1987; Scliar-Cabral, Nepomuceno e Morais, em preparação), parece valer a pena investigar se os repentistas são melhores leitores do que os não-repentistas. Diante dos trabalhos sobre a relação entre a repetição e as confusões na reprodução de rimas (Conrad, 1971; Hulme, 1984), nós também escolhemos observar se os repentistas seriam ou não melhores que os não-repentistas em tarefas de memória auditiva. Em resumo, nós estávamos interessados em investigar se a aptidão para rimar é uma habilidade independente ou se está fortemente interligada com outras habilidades.

\section{Metodologia}

\section{SUJEITOS}

A amostra investigada foi formada por 38 sujeitos do sexo masculino, de nível sócio-econômico baixo, sendo a maioria do Estado de Pernambuco. Deles, 20 eram repentistas e 18 eram não-repentistas, mas de um contexto sócio-educacional semelhante. Os repentistas eram, em média, um pouco mais velhos do que os não- 
repentistas (a média de idade dos repentistas era 45,1 anos e a dos nãorepentistas era de 32,3).

A média percentual em um teste de inteligência não-ver-bal INV (vide tarefa 5, abaixo) - foi de 23,1 para os repentistas e 41,41 para os não-repentistas; esta diferença foi significativa $[F(1,36)=4,40 ; p<0,05]$. $\mathrm{O}$ escore bruto parece ser uma medida mais apropriada que o percentil, já que esta última medida foi provavelmente obtida pela padronização do teste em populações bastante diferentes das que estão sendo estudadas aqui. $\mathrm{O}$ escore médio obtido neste teste pelos repentistas foi de 23,4 e o obtido pelos não-repentistas foi de 32,9. Mais uma vez, esta diferença em favor dos não-repentistas foi significativa $[F(1,36)=7,06$; $<<0,05]$.

Por causa dessa diferença significativa no escore bruto do teste INV, como também pela diferença de idade entre os dois grupos de sujeitos, foi decidido, ao ser testada a significância das diferenças dos grupos no desempenho das tarefas discutidas abaixo, fazer-se uma análise de covariância, considerando como cova-riadas as variáveis idade e escore bruto no INV.

\section{PROCEDIMENTO}

Os dados foram coletados através de testes individuais. Cada pessoa era examinada em entrevistas previamente combinadas, em casa, em um lugar onde as tarefas pudessem ser executadas sem risco de perturbação. Foram realizadas duas sessões, cada uma com a duração de cerca de uma hora e meia. No começo, a pessoa era entrevistada acerca de detalhes da sua experiência educacional, que, na maioria dos casos, não tinha seguido um caminho convencional, contínuo, mas fragmentado e interrompido.

As tarefas dadas foram as seguintes:

a) Produção de Rimas: O experimentador solicitou a cada pessoa que produzisse tantas rimas quantas pudesse, depois de ouvir uma palavra-estímulo. As instruções foram: "Eu gostaria que você fizesse rimas. Eu vou dizer uma palavra e você deverá dizer rapidamente tantas rimas quantas puder."

R. bras. Est. pedag., Brasília, v.72, n.172, p.291-317, set./dez. 1991 
Foram apresentadas ao sujeito três séries - concreta, abstrata e sem sentido - , cada qual consistindo de seis palavras-estímulo. Antes das tentativas experimentais em cada série, alguns exemplos foram dados para se assegurar de que as tarefas eram claramente entendidas. A ordem de apresentação das séries foi controlada. $\mathrm{O}$ experimentador mediu o tempo gasto pelo sujeito para produzir a primeira rima e, depois, permitiu que o sujeito não demorasse mais de 150 segundos para produzir as outras.

b) Segmentação Fonológica - Adição e Deleção: Este teste, semelhante ao usado por Morais, Cary, Alegria e Bertelson (1979), foi aplicado em duas versões: uma envolvendo adição e ou tra envolvendo deleção. A tarefa de deleção envolvia a subtração do primeiro fonema de uma palavra $(\mathrm{P})$ ou não-palavra $(\mathrm{N})$ dada pelo experimentador. A tarefa de adição exigia a adição de um fo nema extra no começo de uma palavra ou não-palavra. A tarefa foi dividida em três sessões: $1^{\text {a }}$ ) envolvendo a transformação de palavras reais em outras palavras reais (PP); $2^{\mathrm{a}}$ ) envolvendo a transformação de não-palavras em outras não-palavras $(\mathrm{NN}) ; 3^{\mathrm{a}}$ ) envolvendo a transformação de não-palavras em palavras reais (NP). Havia 30 itens em cada sessão. Além disso, cada sessão in cluía um pré-teste constituído de 21 tentativas. Assim, cada tare fa consistia de 90 tentativas propriamente ditas e 63 tentativas de pré-teste. Foi usado um procedimento de correção durante as tentativas do pré-teste. Quando a pessoa testada errava, o experi mentador fornecia as respostas corretas. A tarefa de adição foi da da a 8 repentistas e 15 não-repentistas, e a tarefa de deleção foi dada a 12 repentistas e 12 não-repentistas. Cada sujeito foi testa do em todos os tipos de transformação (PP:NN:NP) e a ordem de apresentação foi controlada.

c) Velocidade de Leitura: Foram apresentadas aos sujeitos 68 palavras impressas em maiúsculas em tipos grandes e organizadas em quatro colunas. Foi-lhes dito: "Eu gostaria de ver quantas palavras você pode ler em um minuto. Por favor, leia estas palavras tão rápido quanto você puder." As palavras eram todas de alta freqüência e de duas ou três sílabas.

d) Memória Auditiva: Foi usado um subteste da bateria 
CEPA. Esse subteste consiste em duas listas - A e B -, cada qual contendo 30 palavras. Depois de ouvi-las ditas pelo examinador, os sujeitos tentavam repetir tantas quantas pudessem, em qualquer ordem.

e) Teste de inteligência não-verbal (INV): Este teste de inteligência não-verbal é semelhante às Matrizes Progressivas de Raven.

\section{RESULTADOS}

a) Produção de Rimas: Serão discutidos aqui dois aspectos dos resultados da tarefa de produção de rimas: o número de rimas produzido e o tempo gasto para produzir a primeira rima.

a.a) Número de rimas produzidas: Como pode ser visto na Tabela 1 e na Figura 1, os repentistas produziram cerca de quatro vezes mais rimas por pessoa do que os não-repentistas. A média total de rimas produzidas por pessoa pelos repentistas para todos os 18 estímulos foi de 114,9 (escore mínimo 67 e escore máximo 144). A média total correspondente produzida por pessoa pelos não-repentistas foi de 27,6 (escore mínimo 6 e escore máximo 49). Desta forma, os repentistas produziram, em média, quatro vezes mais rimas por pessoa do que os nãorepentistas. Os repentistas, além de produzirem um número muito superior de rimas do que os não-repentistas, cometeram também menos erros: cerca de 98\% das respostas dos repentistas, contra apenas 36\% das dos não-repentistas, eram rimas corretas.

Em ambos os grupos, palavras-estímulo concretas provocavam mais rimas do que palavras-estímulo abstratas, que, por

Tabela 1 - Número médio de rimas produzidas por pessoa

\begin{tabular}{|c|c|c|c|}
\hline \multirow[t]{2}{*}{ Grupo } & \multicolumn{3}{|c|}{ Tipos de Palavras-Estímulo } \\
\hline & Concreta & Abstrata & Sem sentido \\
\hline Repentistas Não- & 50.310 .8 & $38.9 \quad 7.9$ & $28.7 \quad 7.9$ \\
\hline Repentistas & $10.8 \quad 6.3$ & $9.5 \quad 5.2$ & $7.3 \quad 5.2$ \\
\hline
\end{tabular}

Desvio padrão em itálico 
sua vez, provocavam mais rimas do que palavras-estímulo sem sentido. As diferenças entre os diversos tipos de palavras eram maiores para os repentistas do que para os não-repentistas. A média de rimas que os repentistas produziram para todas as seis palavras-estímulo concretas foi de 50,4; para as seis palavras-estímulo abstratas e as seis palavrasestímulo sem sentido, esta média foi, respectivamente, de 35,9 e 28,7. As médias correspondentes para os não-repentistas foram, respectivamente, de 10,8, 9,5 e 7,3. Desta forma, os repentistas produziam cerca de cinco vezes mais rimas por pessoa em palavras abstratas, aproximadamente quatro vezes mais rimas por pessoa em palavras concretas e quase exatamente quatro vezes mais rimas por pessoa em palavras sem sentido do que os não-repentistas.

Para verificar o nível de significância das diferenças entre as médias, foi realizada uma análise de covariância mista com medidas repetidas na condição tipo de palavras-estímulo (3: palavras concretas versus palavras abstratas versus palavras sem sentido), tendo a idade e o QI como covariadas. O Grupo (2: repentistas e não-repentistas) foi o fator entre-sujeitos. A diferença entre o número de rimas produzidas pelos repentistas e pelos não-repentistas teve de fato alta significância $[F(1,34)=$ $159,65 ; \mathrm{p}<0,001]$. As diferenças entre as condições (palavras concretas versus palavras abstratas versus palavras sem sentido) eram também significativas $[\mathrm{F}(2,72)=49,50 ; \mathrm{p}<0,001]$. Uma análise estatística $a$ posteriori (Teste de Tukey) mostrou uma diferença significativa entre o número de rimas produzidas entre palavras concretas e palavras abstratas $(p<0,01)$, entre palavras sem sentido e palavras abstratas $(p<0,01)$ e entre palavras sem sentido e palavras concretas $(p<0,01)$. Foi encontrada também uma interação significativa entre grupo versus condição $[\mathrm{F}(2,72)=26,69 ; \mathrm{p}<0,001]$. Uma análise estatística a posteriori (Teste de Newman-Keuls) mostrou que os repentistas diferiam significativamente dos não-repentistas no que se refere ao número de rimas produzidas diante das palavras concretas $(p<0,01)$, das palavras abstratas $(p<0,01)$ e-das palavras sem sentido $(p<0,01)$. As diferenças entre as condições concreta e abstrata, concreta e sem sentido e abstra- 
ta e sem sentido eram significativas $(p<0,01)$ para os repentistas, mas não para os não-repentistas.

\section{FIGURA 1 \\ NUMERO MEDIO DE RIMAS PRODUZIDAS}

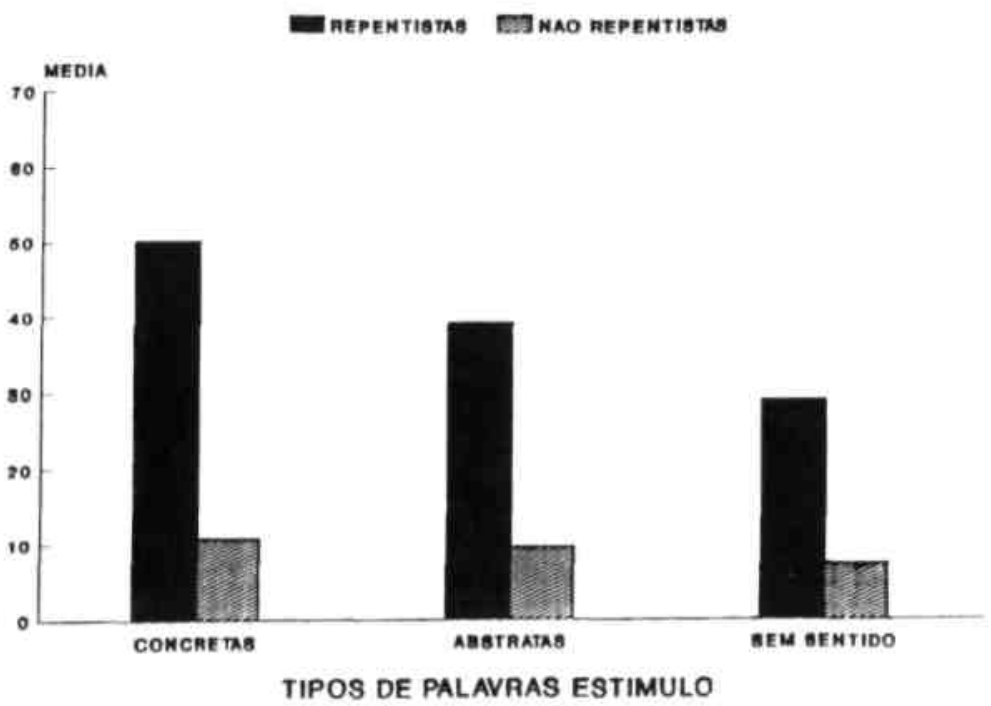

b) Tempo Gasto para Produzir a Primeira Rima: A Tabela 2 mostra que os repentistas eram também mais rápidos em produzir a primeira rima que os não-repentistas. $\mathrm{O}$ tempo médio para produzir a primeira rima foi de 20,3 segundos para os repentistas e de 56,1 segundos para os não-repentistas. Desta forma, a razão entre o tempo gasto pelos repentistas e pelos não-repentistas era de 2,8:1. O tempo médio para produzir a primeira rima para palavras concretas foi de 13,2 segundos para os repentistas e de 62,3 segundos para os não-repentistas (razão 4,7:1). No caso de palavras-estímulo abstratas, a média foi de 15,5 segundos para os repentistas e de 57,3 segundos para os não-repentistas (razão 3,7:1). Para palavras-estímulo sem sentido, a média foi de 32,1 segundos para os repentistas e de 48,6 segundos para os não-re-

R. bras. Est. pedag., Brasília, v.72, n.172, p.291-317, set/dez. 1991 
pentistas (razão 1,5:1) — ver Figura 2. Os repentistas eram mais vagarosos ao produzirem rimas diante de palavras sem sentido do que diante de outros tipos de palavras, enquanto que o contrário era verdadeiro para os não-repentistas.

Tabela 2 - Tempo médio em segundos gasto por pessoa para produzir a primeira rima

\begin{tabular}{|c|c|c|c|c|c|}
\hline \multirow[t]{2}{*}{ Grupo } & \multicolumn{5}{|c|}{ Tipos de Palavras-Estímulo } \\
\hline & Conc & & $\mathrm{Abs}$ & & Sem sentido \\
\hline Repentistas Náo- & $\begin{array}{l}13.2 \\
62.3\end{array}$ & $\begin{array}{r}4.8 \\
28.0\end{array}$ & 15.6 & 5.1 & $32.1 \quad 17.2$ \\
\hline Repentistas & & & 57.3 & 37.9 & $\begin{array}{ll}48.6 & 39.2\end{array}$ \\
\hline
\end{tabular}

Desvio padrão em itálico

Mais uma vez, a diferença entre os dois grupos (repentis-tas versus não-repentistas) é evidente, a partir dos dados brutos. Para verificar o nível de significância das diferenças entre essas médias foi realizada uma análise de covariância mista com medidas repetidas na condição tipo de palavras-estímulo (3: palavras concretas versus palavras abstratas versus palavras sem sentido), tendo a idade e o QI como covariadas. O Grupo (2: repentistas e não-repentistas) foi o fator entre-sujeitos. A diferença entre os grupos foi altamente significativa $[F(1,34)=22,64$; $\mathrm{p}<0,001]$. Não houve nenhum efeito principal significativo do tipo de palavra $[\mathrm{F}(2,72)=1 ; \mathrm{p}=$ n.s.], mas a interação do grupo versus tipo de palavras foi altamente significativa $[\mathrm{F}(2,72)=18,31 ; \mathrm{p}<0,001]$. $O$ teste aposteriori de Newman-Keuls mostrou uma diferença significativa $(p<0,01)$ entre os repentistas e os não-repentistas, para cada uma das três condições. Foram também significativas $(p<0,01)$ as diferenças entre $o$ tempo médio gasto pelos repentistas para produzirem as primeiras rimas diante de palavras abstratas versus palavras sem sentido e de palavras concretas versus palavras sem sentido, mas não houve significância na diferença entre o tempo médio que eles gastaram para produzir a primeira rima di- 
ante de palavras concretas versus palavras abstratas. Os não-re-pentistas diferiam significativamente $(\mathrm{p}<0,05)$ em relação ao tempo médio gasto para produzirem a primeira rima diante de palavras abstratas versus palavras sem sentido e palavras concretas versus palavras sem sentido, mas não em relação ao tempo médio gasto para produzirem a primeira rima diante de palavras concretas versus palavras abstratas.

\section{FIGURA 2 TEMPO MEDIO EM SEGUNDOS GASTO POR PESSOA PARA PRODUZIR A PRIMEIRA RIMA}

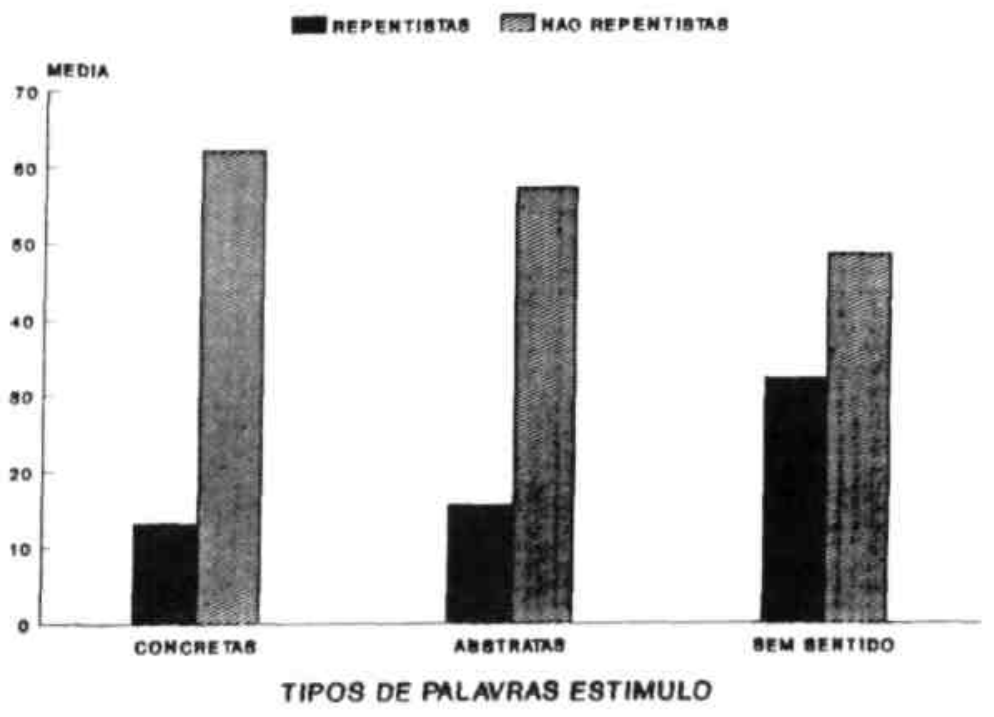

c) Segmentação Fonológica: O escore médio dos repentis-tas nessa tarefa foi de 82,75 (d.p. 6,99), de um total de 90 , e o dos nãorepentistas foi 82,05 (d.p. 11,32). Assim, os escores dos dois grupos foram quase idênticos. É necessário algum cuidado ao interpretar este resultado, porque um efeito de quase-teto pode ter obscurecido as reais diferenças entre os grupos. De fato, este efeito de teto torna desnecessária a análise estatística da tarefa de 
segmentação fonológica. Uma descrição mais detalhada do desempenho dos sujeitos nesta tarefa é apresentada na Tabela 3.

\begin{tabular}{|c|c|c|c|c|c|c|}
\hline \multirow[t]{2}{*}{ Grupo } & \multicolumn{3}{|c|}{ Adição } & \multicolumn{3}{|c|}{ Deleção } \\
\hline & PP & NP & $\mathrm{NN}$ & PP & NP & $\mathrm{NN}$ \\
\hline Repentistas Não- & $\begin{array}{l}24.1 \\
6.1 \\
24.2 \\
4.1\end{array}$ & $\begin{array}{r}29.0 \\
1.2 \\
29.0 \\
1.2\end{array}$ & $\begin{array}{r}25.4 \\
4.4 \\
23.2 \\
9.6\end{array}$ & $\begin{array}{r}28.8 \\
1.7 \\
27.9 \\
3.2\end{array}$ & $\begin{array}{r}29.2 \\
1.3 \\
29.3 \\
2.0\end{array}$ & $\begin{array}{r}27.7 \\
3.0 \\
27.2 \\
4.5\end{array}$ \\
\hline Repentistas & & & & & & \\
\hline
\end{tabular}

PP = Palavra-Palavra; NP = Não-Palavra-Palavra; NN = Não-Palavra-Náo-Palavra Desvio padrão em itálico

d) Velocidade de Leitura: O número médio de segundos gasto por pessoa para ler uma palavra era 1,4, no caso dos repen-tistas, e 1,5, no caso dos não-repentistas. Assim, havia pouca diferença na velocidade de leitura entre os repentistas e os não-repentistas. Para analisar esta conclusão estatisticamente, foi efetuada uma análise simples de covariância entre os grupos, com a idade e o QI como covariados. A diferença entre os grupos não foi significativa $[F(1,34)=0,81 ; p=n . s$.$] .$

e) Memória Auditiva: Os repentistas recordaram uma média de 12,7 palavras por pessoa, enquanto que os não-repentistas recordaram uma média de 12 palavras. Assim, os repentistas e os não-repentistas não diferiram no que se refere à memória imediata de palavras. Para analisar esta conclusão estatisticamente, foi feita uma análise simples de covariância entre os grupos, com o QI e a idade como covariadas. A diferença entre os grupos não foi significativa $[F(1,34)=1,55 ; p=n . s]$.

\section{Discussão}

A descoberta mais evidente deste estudo é que os repentistas têm um desempenho muito melhor do que os não-repen- 
tistas em tarefas de rimas. Era de se esperar que eles fossem rimadores mais prolixos, mas a extensão de sua superioridade foi marcante. Em média, eles produziram cerca de três vezes mais rimas que os nãorepentistas.

Contudo, eles não diferiram significativamente dos nãorepentistas em relação à velocidade de leitura, à memória auditiva (pelo menos enquanto medida pela habilidade de reproduzir listas de palavras ou palavras isoladas) ou segmentação fonológica. Pelo contrário, eles desempenharam um pouco pior que os não-repentistas em um teste de QI envolvendo habilidade espacial (embora essa diferença pareça estar, sobretudo, relacionada ao fato de eles serem mais velhos).

Assim, este estudo é uma história de dissociação: dissociação entre rima e certas aptidões lingüísticas; dissociação entre ensino formal e o desenvolvimento de habilidades informais valorizadas culturalmente e, o mais importante, uma dissociação ainda mais ampla que ocorre entre habilidades isoladas e aptidões, de tal forma que, por exemplo, uma habilidade marcante pode, como acontece com a aptidão dos repentistas para a rima, se desenvolver independentemente de outras aptidões.

Neste estudo, a maior aptidão dos repentistas para a rima não está relacionada à sua superioridade na leitura. É necessário tomar cuidado ao interpretar os dados relativos à segmentação fonológica, em virtude de um efeito de quase-teto. Seria desejável investigar se os repentistas e os não-repentistas diferem completamente em tarefas mais complexas que envolvem a manipulação de fonemas (por exemplo, inversões).

Entretanto, os resultados parecem estar de acordo com pontos de vista como os de Bryant, MacLean, Bradley e Crossland (1990), de que a rima não deveria ser vista simplesmente como um sinal da consciência fonológica. Ela é uma capacidade especial que pode ocorrer independentemente de outros aspectos da consciência fonológica. Ainda está para ser verificado até que ponto isto também pode ser verdadeiro para outras aptidões fonológicas específicas. Diante dos fatos de que Morais e seus colegas (Cary, Morais e Bertelson, 1987; Morais, 1988) descobriram dois poetas 
analfabetos portugueses que eram altamente sensíveis à aliteração; de que Dowker (1989) descobriu que a aliteração é freqüentemente um fato na poesia de crianças muito pequenas, e de que Bryant, MacLean, Bradley e Crossland (1990) obtiveram resultados sugerindo que a sensibilidade à aliteração seria um melhor preditor da capacidade de leitura do que a sensibilidade à rima, parece que seria altamente desejável investigar se os repentistas são superiores aos não-repentistas na sua habilidade para usarem a aliteração. Se eles o forem, isso implicaria que os repentistas têm um aumento generalizado da capacidade de produzir palavras que sejam fonologicamente relacionadas a palavras dadas, e que isto não é verdade somente para um determinado tipo de relação sonora para a qual eles tenham sido treinados (a aliteração não é um artifício usado pelos repentistas no Brasil).

Outro aspecto interessante deste estudo é que não só os nãorepentistas produziram significantemente menos rimas corretas do que os repentistas como muitos deles, ao contrário dos repentistas, também produziram uma boa qualidade de respostas incorretas. Mais ainda: muitas dessas respostas incorretas eram assonâncias ou aliterações produzidas em resposta ao pedido de rimas. Isso pode"sugerir que a experiência dos repentistas para compor poesia rimada pode residir não só no fato de possuírem uma elevada capacidade de produção de rima mas também em uma elevada consciência do que não é uma rima.

Uma outra diferença entre o desempenho para rimar dos repentistas e dos não-repentistas é que, enquanto os primeiros produziam mais rimas e eram mais rápidos para produzirem a primeira rima diante de palavras concretas, e produziam menos rimas e eram mais lentos ao produzirem a primeira rima quando lhes eram apresentadas palavras sem sentido, os não-repentistas não mostravam tais diferenças em um grau tão elevado. Na verdade, quando o critério era o tempo gasto para produzir a primeira rima, os não-repentistas tinham um desempenho melhor diante de palavras sem sentido do que de outros tipos de palavras. É difícil, neste momento, interpretar essa diferença; mas parte da explicação deve residir no fato de que os repentistas, no seu coti- 
diano, são freqüentemente solicitados a dar rimas para palavras-estímulo concretas, menos freqüentemente para palavras-estímulo abstratas e quase nunca para palavras-estímulo sem sentido, enquanto que tais diferenciais na experiência não afetariam os não-repentistas, que raramente são solicitados a produzir qualquer tipo de rima.

O desenvolvimento de uma habilidade verbal altamente sofisticada em um grupo de pessoas com escolarização limitada é uma evidência de que a escola formal não é o único caminho através do qual tais habilidades podem ser adquiridas e que as pessoas com pouca ou nenhuma escolaridade podem ter um desempenho muito bom em tarefas intelectuais que sejam apropia-das aos requisitos de sua própria cultura ou ocupação. O Labora tory of Human Cognition (1983) afirma que "as pesquisas realizadas na última década sugerem fortemente que não há limites para o contexto do comportamento, que é muitas vezes pensado em termos gerais". Por exemplo, Cole, Gay e Glick (1974) descobriram que os adultos liberianos analfabetos tinham um desempenho melhor que os americanos adultos escolarizados em uma tarefa que envolvia estimar o número de xícaras de arroz contidas em uma tigela (o arroz é a principal colheita daquele povo). Por outro lado, os liberianos tinham um desempenho relativamente pobre (pior que o das crianças escolarizadas) na identificação de certas figuras geométricas abstratas, tarefa que ocupa uma parte das lições escolares de matemática, mas que tem um papel muito restrito na vida dos liberianos adultos.

Foi sugerido (Scribner e Cole, 1973) que o aprendizado informal difere do escolar por ser o primeiro restrito a situações específicas, enquanto que o segundo é aplicável a uma grande variedade de situações. Resultados de pesquisas recentes de Nunes (por exemplo, Carraher, 1986; Nunes', 1991) têm, contudo, indicado que pelo menos algumas habilidades adquiridas informalmente podem ser transferidas a outros contextos diferentes daqueles em que foram aprendidas. Não obstante, parece que, freqüentemente, 
tanto o aprendizado formal como o informal são ambos referentes a um contexto especificó. Os meninos camelos brasileiros, que são capazes de efetuar cálculos numéricos necessários às suas transações comerciais na feira, têm um desempenho muito pior quando são solicitados a efetuar os mesmos cálculos em uma situação escolar formal. Lave, Murtagh e Rocha (1984) descobriram que, mesmo americanos altamente escolarizados resolvem problemas aritméticos muito melhor em um contexto informal de compra que em um contexto formal escolar. Por outro lado, Ginshurg (1977), Hart (1981) e outros têm dado muitos exemplos de crianças que aprendem procedimentos de cálculos ensinados nas escolas como rotinas que elas não parecem compreender e que não são capazes de transferir para situações informais.

Até em tarefas piagetianas os tipos de contexto de exame, formal e informal, são ambos referentes a contextos específicos. Por exemplo, Roazzi (Roazzi, 1986; Ceei e Roazzi, 1992) encontrou crianças vendedoras de balas na rua de bairros pobres, com insucesso na prova piagetiana de inclusão de classes apresentada de maneira formal, que eram capazes de resolver essa prova em um contexto cotidiano (interação comprador-vendedor). Nesse contexto de compra e venda, contexto mais significativo para essas crianças, a dificuldade encontrada na tarefa tradicional clássica de inclusão de classes desaparecia. Mais recentemente, Roazzi (Roazzi, 1989; Roazzi e Bryant, 1992) tem mostrado como as diferenças entre classes sociais na tarefa piagetiana de conservação desaparecem de forma dramática quando o contexto de comunicação entre o sujeito e o experimentador é controlado de forma a eliminar possíveis "pistas enganadoras" que poderiam levar as crianças a interpretações erradas sobre o que fazer.

$\mathrm{O}$ desenvolvimento relativamente independente de certas habilidades específicas não é, contudo, confinado a pessoas com uma escolaridade formal limitada ou a habilidades que são diferentemente enfatizadas por diferentes grupos culturais ou ocupacionais. Há muitos exemplos de pessoas que desenvolvem especializações em uma determinada área, não extensíveis a outras áreas. Ericsson e Chase (1981) descreveram um não-graduado que 
praticava a memorização de cadeias de dígitos durante uma hora por dia, durante um período de mais de dois anos. $\mathrm{O}$ seu rol de dígitos aumentou de sete para mais de 80 dígitos. Sua habilidade, contudo, permanecia limitada a dígitos; para outros tipos de estímulos, tais como consoantes aleatórias, ele continuava tendo uma capacidade de memória de cerca de sete itens.

Mesmo exemplos mais extremos de desenvolvimento específico de aptidões cognitivas que são independentes de outras aptidões ou da inteligência geral podem ser descobertos em idiots savants, pessoas mentalmente deficientes que demonstram uma habilidade particular de alto nível (Hermelin e 0'Connor, 1983; 0'Connor e Hermelin, 1984; Shuter-Dyson, 1982; Sloboda, Hermelin e 0'Connor, 1985). Por exemplo, um calculador de calendário pode ser capaz de dizer rapidamente o dia da semana no qual caiu ou cairá uma data particular, enquanto é incapaz de resolver simples cálculos aritméticos. Conforme notaram 0'Connor e Hermelin, a existência de tais pessoas parece colocar problemas para uma teoria unitária da inteligência como a de Spearman (1927) e dar algum suporte a teorias como as de Thurstone (1938), Guilford, (1956) e Gardner (1983), que postularam um conjunto de múltiplas aptidões, independentes ou parcialmente independentes.

Resumindo: neste estudo a especialização dos repentistas estava associada à sua habilidade, muito superior, para rimar, mas não a qualquer outra aptidão não testada neste estudo - por exemplo, memória auditiva para prova contínua, ou para versos, mais do que para listas de palavras. É particularmente interessante verificar se os repentistas seriam mais capazes que os não-repentistas de recordar material poético, dado o fato de que a rima é usada em mnemônicas para ajudar a recordar. Neste sentido, Wallace e Rubin (1988) descobriram que não-graduados eram mais aperfeiçoados em recordar versos de baladas que continham muitos artifícios poéticos, tais como aliteração e assonância, e onde a informação rítmica era enfatizada. No que se refere à leitura, será que a experiência de ser um repentista tem efeitos sutis nas formas como as pessoas lêem? Tenderiam as pessoas a ser - 
na terminologia de Baron, Treiman, Wilt e Kellman (1980) - mais "chinesas" (tendendo a ir diretamente do impresso para o significado) ou mais "fenícias" (tendendo a seguir de grafema para fonema, decodificando regras) nas suas abordagens da leitura?

A superioridade específica dos repentistas em rimar, em relação aos não-repentistas, também levanta a questão se os repentistas teriam um melhor desempenho em tais tarefas, do que pessoas de classe média com uma educação formal mais extensa.' Embora nenhuma pessoa que participou deste estudo seja totalmente iletrada, a maioria tinha uma escolaridade limitada. Será que a escolarização e a experiência de ser um repentista não são simplesmente caminhos alternativos para alcançar o tipo de consciência lingüística necessária à aptidão para produzir rimas? Ou será que a aptidão dos repentistas é uma habilidade específica altamente desenvolvida, que não pode ser substituída pelo treino formal de linguagem, do tipo que é dado na escola? A nossa previsão é de que uma escolaridade mais extensa levaria a uma melhoria na aptidão para rimar, de tal forma que os não-repentistas de classe média se sairiam melhor nesta tarefa do que os não-repentistas de baixo nível sócio-econômico, mas ainda assim teriam um desempenho muito pior do que os repentistas com muito menor nível de escolaridade formal.

Referências Bibliográficas

BADDLEY, A. The psicology of memory. New York: Basic Books, 1978.

BARON, J., TREIMAN, R, WILT, J.F., KELLMAN, P. Spelling and reading by rules. In: FRITH, U. (Ed.). Cognitive processes in spelling. London: Academic Press. 1980.

BEECH, J., COLLEY, A. (Eds.). Cognitive approaches to reading. Chichester: J. Wiley, 1987.

BERTELSON, P., GELDER, B. de, TFOUNI, L.V., MORAIS, J. Metaphonological abilities of adult illiterates: new évidence of heterogeneity. European Journal of Cognitive Psychology, n.1, p.239$250,1989$. 
BRADLEY, A., BRYANT, P. Categorizing sounds and learning to read: a causal connection. Nature, n.301, p.419-421, 1983.

BRUCE, D.J. The analysis of word sounds. British Journal of Educational Psychology, v.34, p. 158-170, 1964.

BRYANT, P., BRADLEY, L. Children's reading problems. Oxford: Basil Blackwell, 1985.

BRYANT, P., MACLEAN, M.E., BRADLEY, L.L., CROSSLAND, J. Rhyme and alliteration, phoneme detection and learning to read. Developmental Psychology, v.26, n.3, p.429-438, 1990.

CARRAHER, T.N. From drawings to building: Working with mathematical scales. International Journal of Behauioural Development, v.9, p.527-544, 1986.

CARY, L., MORAIS, J., BERTELSON, P. A case study: the metaphonological abilities of an illiterate-poet. [S.Ls.n.], 1987. Pôster apresentado no II Meeting of the European Society of Cognitive Psychology, Madrid, Sept. 1987.

CECI, S., ROAZZI, A. The effects of context on cognition: portraits from Brazil. In: STERNBERG, R. (Ed.). Mind in context: mteractionist perspectives on human lntelligence. Cambridge: Cambridge University, 1992.

CHUKOVSKY, K. From two to five. Trad. por Mirian Morton. Berkeley: University of Califórnia, 1968. Original russo. Traduzido da edição de 1959.

COLE, M., GAY, J., GLICK, J. Some experimental studies of Kpelle quantitative behaviour. In: BARRY, J.W., DASEN, P.R (Eds). Culture and cognition: reading in cross-cultural Psychology. London: Methuen, 1974.

CONRAD, R. The cronology of development of covert speech in children. Developmental Psychology, v.5, p.398-405, 1971.

DOWKER, A.D. Rhyme and alliteration in poems elicited from young children. Journal of Child Language, v.16, p.181-202, 1989. 
ERICSSON, K., CHASE, W.G. Skilled memory. In: ANDERSON, J.R. (Ed.). Cognitive skills and their aquisition. Hillsdale: Lawrence Erlbaum, 1981.

FINNEGAN, R. Oral poetry. Cambridge: Cambridge University, 1977.

FINNEGAN, R. The penguin book of oral poetry. Harmondsworth: Alan Lane. 1978.

GARDNER, H. Frames of mind: the theory of múltiple lntelligence. London: Heinemann, 1983.

GINSBURG, H.P. Children's arithmetic. New York: Van Norstrand, 1977.

GUILFORD, J.P. The structure of intellect. Psychological Bulletm, v.53, p.267293, 1956

HART, K.M. Childrens understanding of mathematics. Oxford: J. Murray, 1981.

HERMELIN, B., 0'CONNOR, N. Idiot savant calendrical calculators: rules and regularities. Psycological Medicine, v.16, p.885-893,1983.

HULME, C. Developmental differences in the effects of acoustic similarity on memory span. Developmental Psychology, v.20, p.650-652, 1984.

LABORATORY OF HUMAN COGNITION. Culture and cognitive development. In: MUSSEN, P. (Ed.). Handbook of child Psychology. New York: J. Wiley, 1983.

LAVE, J., MURTAGH, M., ROCHA, O. de la. The dialectic of arithmetic in grocery shopping. In: ROGOFF, B., LAVE, J. (Eds.). Everday cognition: it's development in social context. Cambridge: Havard University, 1984.

LENEL, J., CANTER, J. Rhymes recognition and phonemic perception in young children. Journal of Psycholinguistic Research, v. 10, p.57-67, 1981. 
LIBERMAN, I.Y., SHANKWEILER, D., LIBERMAN, A.M., FOWLER, C, FISHER, W.F. Phonetic segmentation and recording in the beginning reader. In: REBER, A.S., SCARBOROUGH, D.A. (Eds.). Towards a Psychology of reading. Hillsdale: Lawrence Erlbaum, 1977.

LUKENS, H.T. Preliminary report on the learning of language. Pedagogical Seminary, v.3, p.424-460, 1984.

LUNDBERG, I., FROST, J., PETERSEN, O. Effects of an extensive program for stimulating phonological awareness in preschool children. Reading Research Quarterly, v.23, p.263-284, 1988.

MACLEAN, M., BRYANT, P.E., BRADLEY, L. Rhymes, nursery rhymes and reading in early Childhood. Merril-Palmer Quarterly, v.33, p.255-282, 1987.

MORAIS, J. Constraints on the development of phonemic awareness. [S.1.: s.n.], 1988. Apresentado no Symposium on Phonological Processes in Literacy to honour the research of Isabelle Y. Liberman, 39th Annual Conference of the Orton Society, Tampa, Nov. 1988.

MORAIS, J. Phonological awareness: a bridge between language and literacy. In: SAWYER, D.Z.J., FOX, B.J. (Eds.). Phonological awareness in reading: the evolution of Current perspectives. Berlin: Springer, 1991.

MORAIS, J., ALEGRIA, J., CONTENT, A. Segmentai awareness: respectable, useful and nearly always necessary. Cahiers de Psychologie Cognitive, v.l, p.530-556, 1987.

MORAIS, J., CARY, L., ALEGRIA, J., BERTELSON, P. Does awareness of Speech as a sequence of the phones arise spontaneously? Cognition, v.l, p.323-331, 1979.

NESDALE, A.R., HERRIMAN, M.L., TUNMER, W.E. Phonological awareness. In: TUNMER, W.E., PRATT, C, HERRIMAN, M.L. (Eds.). Metalinguistic awareness in children: theory research and implications. Berlin: Springer, 1984. 
NUNES, T. Système alternatifs de connaissances selon différents environnements. In: GARNIER, C, BEDNARZ, N., ULANOVSKAYA, I. (Eds.). Après Vygotski et Piaget: perspectives sociale et constructiviste écoles russe et occidentale. Bruxelles: De Bceck Université, 1991.

0'CONNOR, N., HERMELIN, B. Idiot savant calendrical calculators: maths or memory? Psychological Medicine, v.14, p.801-806, 1984.

ROAZZI, A. Implicações metodológicas na pesquisa transcultural: a influência do contexto social em tarefas lógicas. Arquivos Brasileiros de Psicologia, v.38, n.3, p.71-91, 1986.

ROAZZI, A. Desenvolvimento cognitivo e pesquisa piagetiana. In: HUTZ, C. (Ed.). Anais do II Simpósio Brasileiro de Pesquisa e Intercâmbio Científico promovido pela ANPEPP — Associação Nacional de Pesquisa e Pós-Graduação em Psicologia, Gramado, abr. 1989. [S.1.: s.n.], 1989.

ROAZZI, A. Explicações causais em desenvolvimento cognitivo: a relação entre consciência fonológica e leitura. In: FRANCO, M.L.P.B., MACEDO, R.M.S. (Eds.). Anais do III Simpósio Brasileiro de Pesquisa e Intercâmbio Científico, Águas de São Pedro, ago. 1990. Sáo Paulo: ANPEPP: PUC/SP, 1991. P. 14-26.

ROAZZI, A. A consciência fonológica avaliada em tarefas de fusão e segmentação fonêmica/silábica como precursora de posteriores habilidades de leitura. [S.1.: s.n.], 1992. Apresentado na Reunião de Cooperação Técnico-científica entre Pesquisadores do Nordeste, promovida pelo INEP, realizada no Centro de Educação da UFPB, João Pessoa, out. 1992.

ROAZZI, A., BRYANT, P.E.B. Social class, context and cognitive development. In: LIGHT, P., BUTTERWORTH, G. (Eds.). Context and cognition: ways of learning and knowing. Herts: Harvester \& Wheatsheaff, 1992.

ROAZZI, A., DOWKER, A. Consciência fonológica, rima e aprendizagem da leitura. Psicologia: Teoria e Pesquisa, v.5, n.1, p.31-55, 1989. 
SCHIEFFELIN, B.B. Talking like birds: sound play in a cultural perspective. In: OCHS, E., SCHIEFFELIN, B.B. (Eds.). Acquiring conversational Competence. London: Routledge \& Kegan Paul, 1983.

SCHUTER-DYSON, R. Musical ability. In: DEUTSCH, D. (Ed.). The Psychology of music. New York: Academic Press, 1982.

SCHWARTZ, J.I. Metalinguistic awareness: a study of verbal play in young children. [S.1.: s.n.], 1977. Trabalho apresentado no Annual Meeting of the American Educational Research Association.

SCLIAR-CABRAL, L., NEPOMUCENO, LA., MORAIS, J. Diferenças de capacidades de apagamento de vogai e de consoante em sujeitos brasileiros: efeito da algabetização. [S.1.: s.n., 199-]. Em preparação.

SCRIBNER, S., COLE, M. Cognitive consequences of formal education. Science, n.182, p.553-559, 1973.

SLOBODA, J.A., HERMELIN, B., 0'CONNOR, N. An exceptional musical memory. Music Perception, v.3, p.155-170, 1985.

SPEARMAN, C. The abilities of man. New York: MacMillan, 1927.

STANOVICH, K.E., CUNNINGHAM, A.M., CRAMER, B.B. Assessing phonological awareness in Kindergarten children: issues of task comparability. Journal of Experimental Child Psychology, v.38, p.175-190, 1984.

TAVARES, B. Cantadores. Olinda: Casa da Criança, 1982.

THURSTONE, L Primary mental abilities. Chicago: University of Chicago, 1938. (Psychometric monographs, 1).

WALLACE, W.T., RUBIN, D.C. "The wreck of the old 97": a real avent remembered in song. In: NEISSER, U., WINOGRAD, E. (Eds.). Remembering reconsidered: ecological and traditional approaches to the study of memory. Cambridge: Cambridge University, 1988.

Recebido em 20 de novembro de 1991 
Antonio Roazzi, doutor em Psicologia, é professor do curso de mestrado em Psicologia da Universidade Federal de Pernambuco (UFPE).

Ann Dowker e Peter E. Bryant são professores do Departamento de Psicologia Experimental da Universidade de Oxford.

This study deals with repentistas: oral poets who are to be found in the Northeast of Brazil, most of whom have had limited schooling. Twenty repentistas and 18 non-repentistas from a similar backgroundwere giventasksinvolvingrhymesproductions,phonological segmention, memory for word list, and reading spped The repentistas produced about four times as many rhymes as did the non-repentistas, and were much quicker to produce the first rhyme; but the two groups did not differ significantly from one another as regards any other task. Thus the ability of the repentistas appears to be both highly developed and highly specialised Theses findings lend support to the view that there may be dissociai io ris between rhyme and phonological segmenta-tion; between formal education and the development of "informal" cul-turally or occupationally valued skills; and more generally, between separate skills or abilities.

Cet article recherche le rapport entre les habilites linguistiqu.es et I'art du "repente", chez les "repentistas" (guitaristes ou chanteurs), poetes oraux trouves aux Nordest du Brésil, beaucoup deux avec une scolarité bien limitée. Vingt "repentistas" et dix-huit non "repentistas" d'àge, scolarité et niveau socio-économique pareils ont été examines dans un ensemble des taches comme production des rimes, segmentai ton phonologique, mémoire de listes de mots et volocité de lecture. Les "repentistas" ont produit un nombre de rimes quatro fois Superieur aux non "repentistas", autre de pésenter une velocité supérieure dans là production de là première rime. Pour les autres taches, il y a eu peu de différence entre les deux groupes. Alors, on considere que 1'habileté des "repentistas" semble être au même temps três développée et specialisée. Ces decouverts confirment le point de vue selon lequel il peut avoir dis8ociotion entre rimes et quelques autres habilites linguistiques comme 
le segmentation phonologique, aussi comme il semble qu'il n'y a pas un rapport direct entre l'éducation formelle et le développement d'habiletés culturellement non formelles.

Investiga là relación entre Ias habilidades lingüisticas y el arte del repente, com sujetos "repentistas" (violinistas, o cantores), poetas orales encontrados en el Nordeste del Brasil, mucho del los cuales tienen una escolaridad limitada. Veinte "repentistas" y dieciocho no "repentistas" con idad escolaridad y nivel socioeconômico semejante se examina-ron en un conjunto de actividades abarcando producción de rimas, seg-mentación fonológica, memória de listas de palabras y velocidad de lec-tura Los "repentistas" produjeron un número de rimas 4 veces superior a los no "repentistas" además de presentar una velocidad superior en là producción de là primera rima En Ias otras tareas, los dos grupos de sujetos no se diferenciaron de modo significativo. Así là habilidad de los repentistas parece ser al mismo tiempo altamente desarrollada y especializada. Estos descubrimientos corroboran el punto de vista de que puedan haber disociaciones entre rima y al algunas otras habilidades lingüísticas como là segmentación fonológica, así como aparentemente no existe una relación directa entre educación formal y el desarrollo de habilidades culturalmente informales. 